\title{
SIMULATION BASED THROUGHPUT ASSESSMENT OF NON-HOMOGENEOUS TRANSFER LINES
}

\author{
Dhouib, K. ${ }^{*}$; Gharbi, A. ${ }^{* *}$ \& Ayed, S. \\ * Mechanical Engineering and Productique Department, École Supérieure des Sciences et Techniques \\ de Tunis, LMSSDT Laboratory, University of Tunis, 5 Av. Taha Hussein, Tunis, Tunisia \\ ** Automated Production Engineering Department, École de Technologie Supérieure, Production \\ System Design and Control Laboratory, University of Québec, 1100 Notre-Dame Ouest, Montréal, \\ Québec, Canada H3C 1K3 \\ E-Mail: karem.dhouib@esstt.rnu.tn; ali.gharbi@etsmtl.ca; souheil.ayed@esstt.rnu.tn
}

\begin{abstract}
This paper proposes a general simulation model to assess the throughput of non-homogeneous transfer lines. The transfer line has no intermediate buffers between adjacent machines. Machines are subject to random operation dependant failures. The transfer line is dedicated to producing only one product type which can be assimilated to an aggregation of various product types. Due to lack in line balancing, the considered transfer line is non-homogeneous so that its machines have different processing times. The purpose of this paper is to evaluate the performance of approximate analytical approaches dealing with non-homogeneous transfer lines. Several production line configurations have been analyzed and statistical tests were carried out to show that approximate approaches generate significant errors and cannot give a good estimate, compared to simulation results, of the throughput of unbuffered, nonhomogeneous transfer lines subject to operation dependant failures.

(Received in February 2008, accepted in September 2008. This paper was with the authors 2 months for 1 revision.)
\end{abstract}

Key Words: Automated Transfer Lines, Homogenization Techniques, Throughput Evaluation, Simulation Modelling

\section{INTRODUCTION}

Several criteria can be considered in classifying discrete manufacturing systems. The production volume is one of the most important criteria. Based on this classification scheme, three main manufacturing system types can be identified: mass production, batch production, and job shops [1]. Mass production systems are considered the most performing manufacturing systems, despite the lack in flexibility. They are composed of several machines with a product-flow layout and frequently dedicated to manufacture a specific product type with high production volume. Mass production systems are often classified in two categories: flow lines which are asynchronous and manually operated, and transfer lines which are synchronous and automated [2, 3]. In this paper we will focus on the operational issue of automatic transfer lines and on the assessment of their throughput which is considered the most important performance measure of mass production systems.

Transfer line operating machines are subject to random failures which are the principal cause of line inefficiency. Based on effective experience, Buzacott mentioned that production lines are subject to operation dependant failures rather that time dependant failures since manufacturing machines do not age while not operating [4]. Effectively, when a machine failure occurs all operating machines are forced down and the upstream ones will be blocked and the down stream ones will be starved. Also and depending on the design phase of the transfer line and the resulting configuration, transfer lines can be said to be homogeneous or 
non-homogeneous. Homogeneous lines have equal machine processing times. However, nonhomogeneous lines are characterized by difference in processing times. The nonhomogeneous character of transfer lines causes as well blocking and starvation of manufacturing workstations and affects line performance.

Most research realized in the past has dealt with homogeneous transfer lines assuming that the considered manufacturing lines are perfectly balanced $[1,2,5]$. In this paper we study non-homogeneous transfer lines with no intermediate buffers and subject to random failures and repairs. We first expose approximate analytical approaches dealing with nonhomogeneous transfer lines. Then, we propose a general discrete event simulation model to assess throughput of unbuffered, unreliable, non-homogeneous transfer lines. We finally evaluate the throughput of thousands of transfer line configurations randomly generated in order to compare simulation results with approximate analytical models dealing with nonhomogeneous transfer lines. From a practical point of view, many non-homogeneous production lines do not include intermediate buffer. In the case of intermediate buffers, these buffers are not necessarily included between all pairs of machines, but between stages (a stage is composed of several machines in series with no intermediate buffer); we have to evaluate the performance of such production stages before evaluating the performance of the entire system [1].

The remainder of this paper is organized as follows: section two provides characteristics of automatic transfer line, notations, and working assumptions. In section three, we present briefly different techniques proposed by Gershwin [6], Dallery et al. [7], Liu and Buzacott [8], and Chen and Yuan [9] dealing with throughput assessment of non-homogeneous transfer lines. Section four proposes a general simulation model describing the effective dynamic and stochastic behavior of automatic transfer lines. Numerical results, statistical tests and comparison studies conducted through thousands of transfer line configurations are given in section five in order to evaluate the performance of the approximate techniques. Finally, observations and conclusions are provided in section six.

\section{TRANSFER LINES DESCRIPTION AND ASSUMPTIONS}

Automatic transfer lines being studied in this paper consists of a sequence of $m$ machines disposed in a product-flow layout connected by a transfer mechanism. No intermediate buffers are included between machines. The transfer line is dedicated to producing one part type which can be an aggregation of various product types. Parts are transported between workstations simultaneously and flow in a sequence from the first machine until it reaches the last machine after which it leaves the transfer line as a finished part. To be processed in each machine, a part requires a deterministic amount of time. Fig. 1 shows a $n$-machine transfer line and their characteristics according to the following notations and assumptions.

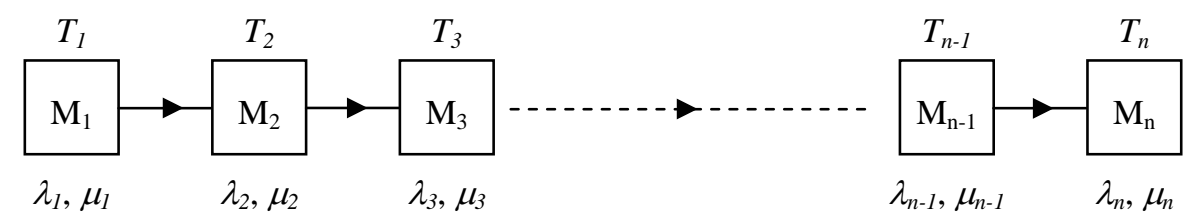

Figure 1: Non-homogeneous $n$-machine transfer line with random failures.

Machines are subject to random failures. When a failure occurs, the upstream machines are blocked, and the downstream ones are starved until the failed machine is repaired. Due to the non-homogeneous character of these transfer lines, blockage and starvation also occur owing to the difference between machine operating times. 


\subsection{Notations}

$n \quad$ - number of machines composing the transfer line.

$i \quad$ - subscript specifying a particular machine number $(i=1,2, \ldots, n)$.

$\mathrm{M}_{\mathrm{i}} \quad-i^{\text {th }}$ machine of the transfer line.

$T_{i} \quad$ - processing time of machine $\mathrm{M}_{i}$.

$T_{F} \quad$ - processing time of line fastest machine : $T_{F}=\min _{i=1}^{n}\left(T_{i}\right)$.

$T_{b o t} \quad$ - processing time of line bottleneck machine : $T_{b o t}=\max _{i=1}^{n}\left(T_{i}\right)$.

$T^{e} \quad$ - processing time of the equivalent homogeneous production line.

$\lambda_{i}\left(\mu_{i}\right)$ - failure (repair) rate of machine $\mathrm{M}_{i}$.

$\operatorname{MTTF}_{i}\left(M T T R_{i}\right)$ - mean time to failure (to repair) of machine $\mathrm{M}_{i}$.

$\lambda_{i}^{e}\left(\mu_{i}^{e}\right)$ - failure (repair) rate of equivalent machine $\mathrm{M}_{i}$ composing equivalent homogeneous production line.

$\lambda_{i 1}, \lambda_{i 2}\left(\mu_{i 1}, \mu_{i 2}\right)$ - failure (repair) rates of equivalent machines $\mathrm{M}_{i 1}$ and $\mathrm{M}_{i 2}$ composing equivalent homogeneous production line proposed by Gershwin [6].

$U T R_{i}$ - steady state availability of machine $\mathrm{M}_{i}$.

UTR - steady state availability of the entire transfer line.

Th - steady state throughput of the entire transfer line.

$T h_{T k} \quad$ - steady state throughput of the entire transfer line evaluated by Technique $k$ (Tk).

$T h_{T k_{-} c}$ - throughput of the $c^{\text {th }}$ line configuration based on Technique $k(\mathrm{Tk})$.

$T h_{s} \quad$ - throughput of production line evaluated by simulation model.

$T h_{S_{-} c}$ - throughput of the $c^{\text {th }}$ line configuration evaluated by simulation model.

$T h \_R E$ - relative error given by comparing analytical throughput to simulation one for all configurations of a $n$-machine line.

$T h \_M R E$ - mean relative error given by comparing analytical throughput to simulation one for all configurations of a $n$-machine line.

\subsection{Working assumptions}

1- Failure times and repair times are exponentially distributed random variables.

2- Failures are operation dependant so that a failure can only occur while the machine is working. Thus, no machine in an up state can break down if it's blocked or starved.

3- Repair actions are done perfectly so the machines are restored to as good as new state.

4- At failure, all machines stop production instantaneously.

5- On failure, parts remain at machines and processing resumes after repair completion.

6- The first station is never starved and the last one is never blocked.

7- Transfer times between machines are considered negligible.

8- No parts are scrapped.

\section{THROUGHPUT ASSESSMENT OF NON-HOMOGENEOUS LINES}

In order to analyse the performance of transfer lines, throughput is often considered as the primary performance measure. In homogeneous case, the throughput of an unbuffered transfer line is simply obtained by multiplying the line availability by its production rate (Eq. 1) [4]; where $1 / T$ is the processing rate of the transfer line.

$$
T h=U T R \cdot \frac{1}{T}
$$


The reliability block diagram of transfer lines can be assimilated to that of series systems since all manufacturing workstations stop producing immediately if any machine breaks down. Hence, the steady state availability of a system with series structure and subject to operation dependant failures can be evaluated by equation (2) [1, 4, 11-14].

$$
U T R=\frac{1}{1+\sum_{i=1}^{n} \frac{\lambda_{i}}{\mu_{i}}}=\frac{1}{1+\sum_{i=1}^{n} \frac{M T T R_{i}}{M T T F_{i}}}
$$

However equation (2) supposes that all machines operate continuously which is the case with homogeneous transfer lines. Nevertheless, with non-homogeneous transfer lines which have different machine processing times; machines have discrete operating behaviour due to the bottleneck one. In this case, blocking and starvation phenomena are not only caused by machine breakdowns, but also due to the non-homogeneous character of such transfer lines. In this section, we present numerous approximation models dealing with throughput assessment of non-homogeneous transfer lines.

\subsection{Gershwin's technique [6]}

Gershwin has proposed a disaggregation technique consisting in decomposing each transfer line machine into two equivalent ones having the same production rate as the fastest machine (F) of the entire transfer line [6]. The first equivalent machine captures the processing time of the original machine and the second one represents its unreliable behaviour (Fig. 2) $\left(T^{e}=T_{F}=\min _{i=1}^{n}\left(T_{i}\right)\right)$.

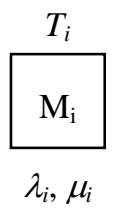

(a) Original machine

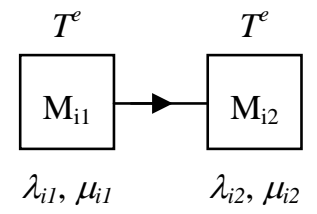

(b) Equivalent machines

Figure 2: Gershwin disaggregation technique.

The equivalent machine parameters were determined by considering that:

1- Throughput of the original machine is equal to that of the two equivalent ones,

2- Repair rate of the original machine is equal to that of the second equivalent one,

3- Throughput without failure of the original machine is equal to that of the first equivalent one,

4- Failure and repair rates of the first equivalent machine are very large compared to those of the second one $\left(\lambda_{i 1}, \mu_{i 1}>>\lambda_{i 2}, \mu_{i 2}\right)$.

\subsection{Dallery et al. technique [7]}

Dallery et al. [7] propose a homogenization technique based on replacing each original machine by an equivalent one (Fig. 3). All equivalent machines have the same processing time which is equal to that of the original line fastest machine $\left(T_{F}\right)$.

The equivalent machine parameters $\lambda_{i}^{e}$ and $\mu_{i}^{e}$ are evaluated by considering that:

1 - The original machine and its equivalent have the same repair process $\left(\mu_{i}^{e}=\mu_{i}\right)$.

2- The isolated throughput of the original machine and its equivalent are equal. 


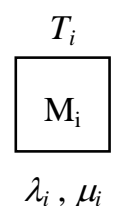

(a) Original machine

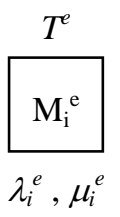

(b) Equivalent machine

Figure 3: Homogenisation technique.

\subsection{Liu and Buzacott's technique [8]}

Liu and Buzacott [8] adopt the same methodology proposed by Dallery et al. [7]. The authors propose four approaches to determine the equivalent machine parameters $\lambda_{i}^{e}$ and $\mu_{i}^{e}$ :

1- The equivalent machine has the same second moment of inter-departure times.

2- The equivalent machine has the same mean down time.

3- The equivalent machine has the same mean up time.

4- The ratio of the production time of the original machine by production time of the equivalent one is an integer.

The authors evaluated the performance of the proposed approaches and found that the first one gives the best estimation of the equivalent machine parameters.

Replacing equivalent machine parameters by their corresponding values in Eq. (2), all three techniques proposed by Gershwin [6], Dallery et al. [7], and Liu and Buzacott [8] give the same analytical formulation of transfer line throughput (Eq. 3).

$$
T H=\frac{1}{T_{\min } \cdot\left(1+\frac{\lambda_{F}}{\mu_{F}}+\sum_{\substack{i=1 \\ i \neq F}}^{n}\left(\frac{\lambda_{i}}{\mu_{i}} \cdot \frac{T_{i}}{T_{\min }}+\frac{T_{i}-T_{\min }}{T_{\min }}\right)\right)}
$$

After conversion and simplification, the throughput of a non-homogeneous transfer line can be evaluated by Eq. (4).

$$
T H=\frac{1}{T_{\min }+\sum_{i=1}^{n}\left(\frac{\lambda_{i}}{\mu_{i}} \cdot T_{i}+T_{i}-T_{\min }\right)}
$$

since for the fastest machine $T_{F}=T_{\min }$, so $\frac{\lambda_{F}}{\mu_{F}}=\frac{\lambda F}{\mu_{F}} \cdot \frac{T_{F}}{T_{\min }}+\frac{T_{F}-T_{\min }}{T_{\min }}$

In the remnant of the paper, T1 refers to the equivalent techniques of Gershwin [6], Dallery et al. [7], and Liu and Buzacott [8]. Hence, the throughput of an $n$-machine transfer line evaluated by Eq. (4) will be referred by $T H_{T 1}$.

\subsection{Chen and Yuan's technique [9]}

In a more recent paper, Chen and Yuan propose to treat the non-homogeneous transfer line approximately as one whose machines have the same production rate which is the smallest production rate among original machines [9]. Hence, the processing times of the equivalent machines are equal to that of the bottleneck machine $\left(T^{e}=T_{\max }=\max _{i=1}^{n}\left(T_{i}\right)\right)$. In this case, no 
modification was introduced to the failure and repair rates of the line original machines. Thus, the transfer line throughput is evaluated by Eq. (1) and its steady state availability can be simply assessed by Eq. (2) with $T$ equals to the processing time of the bottleneck machine. In remainder of the paper, T2 refers to Chen and Yuan technique.

In the next section, we propose a general simulation model imitating the real dynamic and stochastic behaviour of automatic transfer lines. The simulation model allows generating effective throughput of transfer lines in order to evaluate the performance of the approximate studied techniques T1 and T2.

\section{NON-HOMOGENEOUS TRANSFER LINE SIMULATION MODEL}

In order to measure the effective throughput of the non-homogeneous automatic transfer lines, a general discrete event simulation model was developed with AweSim/VisualSlam system [15]. The simulation model imitates the real dynamic and stochastic behaviour of such lines. The simulation model can be used with transfer lines having any length, production times, and failure and repair rates. The flow chart of the simulation model is given at Fig. 4 with the following description of principal modules:

1- The INITIALIZATION module sets for each experiment the number of line machines, the processing time, the mean time to failure $(M T T F)$, and the mean time to repair (MTTR) for each machine. The simulation horizon and the warm up period after which statistics are cleared are also assigned at this step.

2- The PRODUCTION module controls the flow of parts through the transfer line. It controls the instant at which a specific part seize a machine and starts manufacturing, and the release instant at which the part finishes manufacturing and undergoes the next manufacturing machine. Blocking and starvation phenomena due to failure occurrence and to non-homogeneous character of the transfer lines are also managed at this module.

3- The FAILURE \& REPAIR modules sample times to failure and repair durations for each machine from their respective probability distributions. These modules allow changes in machine state from up (down) to down (up) states.

4- The FAILURE INSTANT CORRECTION module adjusts for each machine the effective instant at which failure will occur according to the operation dependant failure (ODF) character of the transfer line (Fig. 5). This module is based on an iterative procedure permitting evaluation of the total idle time recorded during the effective life time of each operating machine.

5- The TIME ADVANCE module uses an algorithm provided by VisualSLAM. It is a discrete event scheduling and a simulation clock which advance the time to the most imminent event (part seizing and releasing a particular machine, failure occurrence at a specific machine, finishing a maintenance action, etc.).

6- The UPDATE PERFORMANCE module saves the number of produced parts during simulation horizon. This allows evaluating the transfer line throughput.

The simulation ends when current simulation time TNOW reaches the simulation horizon.

The effective throughput given by the simulator is compared to that given by techniques $\mathrm{T} 1$ and $\mathrm{T} 2$ through the student's t-test with the following $\mathrm{H}_{0}$ hypothesis:

$\mathrm{H}_{0}$ : the throughput given by analytical formula $\left(T h_{T k}\right)$ is equal to the effective one generated by simulation model $\left(T h_{s}\right)$. 


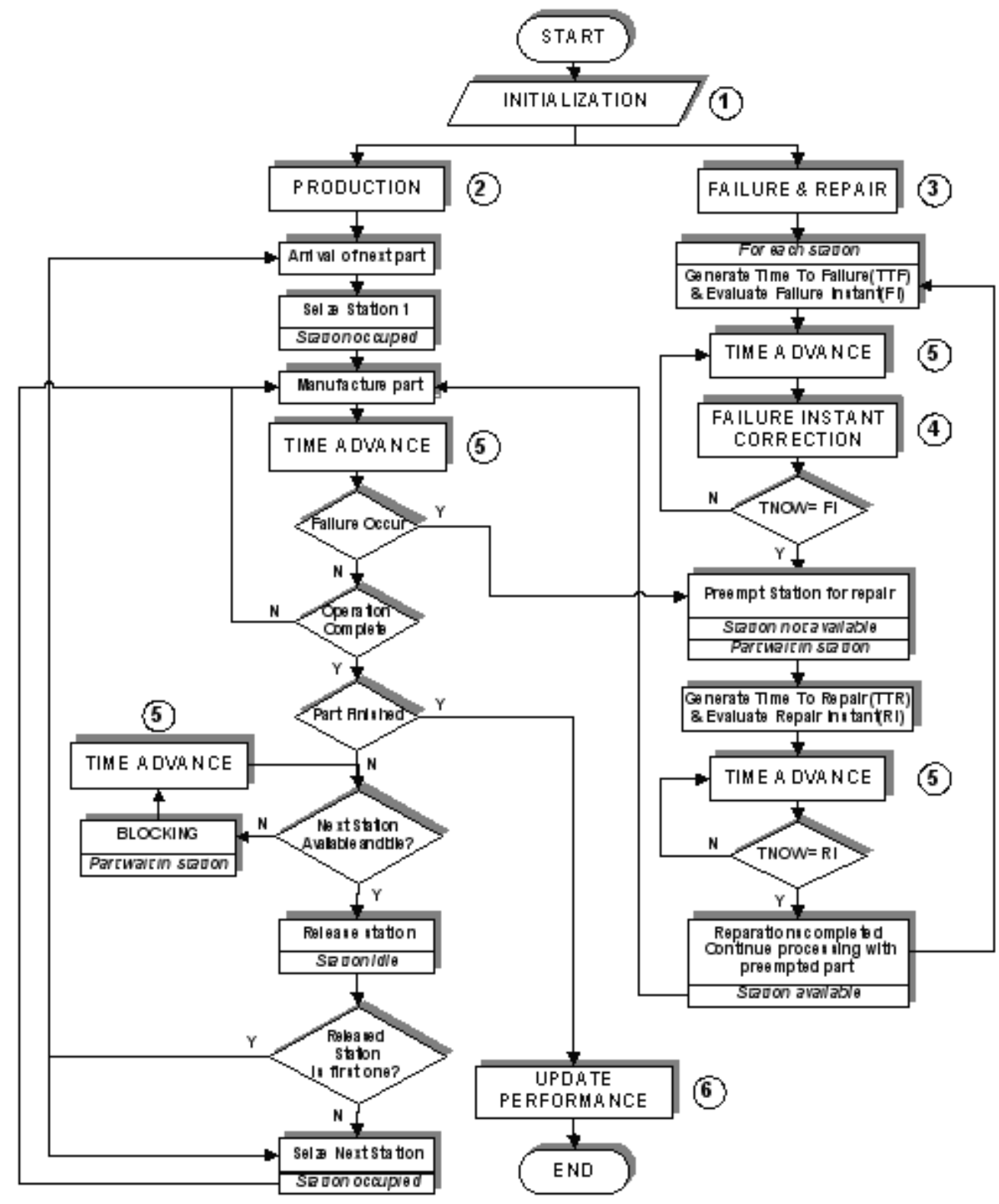

Figure 4: Discrete event simulation model of a general $n$-machine transfer line.

For each line configuration, $N$ replications are performed to obtain $N$ incurred throughput observations. For 1- $\alpha$ confidence level, $\mathrm{H}_{0}$ is rejected if the value of the $t$-distribution with $v$ degrees of freedom and a confidence level 1- $\alpha$ (i.e., $t_{v, 1-\alpha / 2}$ for two sided tests) is greater than the absolute value of $t_{0}$ given by Eq. (5).

$$
t_{0}=\frac{\overline{T h_{s}}-T h_{T k}}{\sqrt{S^{2} / N}}
$$

where : $v=N-1, \quad \overline{T h_{s}}=(1 / N) \cdot \sum_{i=1}^{N} T h_{s}^{i}, \quad S^{2}=(1 /(N-1)) \cdot \sum_{i=1}^{N}\left(T h_{s}^{i}-\overline{T h_{s}}\right)^{2}$

Each simulation is executed during 2.600.000 time units with 100.000 time units of warmup period to guarantee the stability of performance measures. 10 replications were realised with each transfer line configuration. So, For $N=10$ and $\alpha=5 \%$, the Student's t-test table gives $t_{9,0.975}=2.262$. Therefore, the Hypothesis $\mathrm{H}_{0}$ is rejected if $\left|t_{0}\right|>2.262$. 


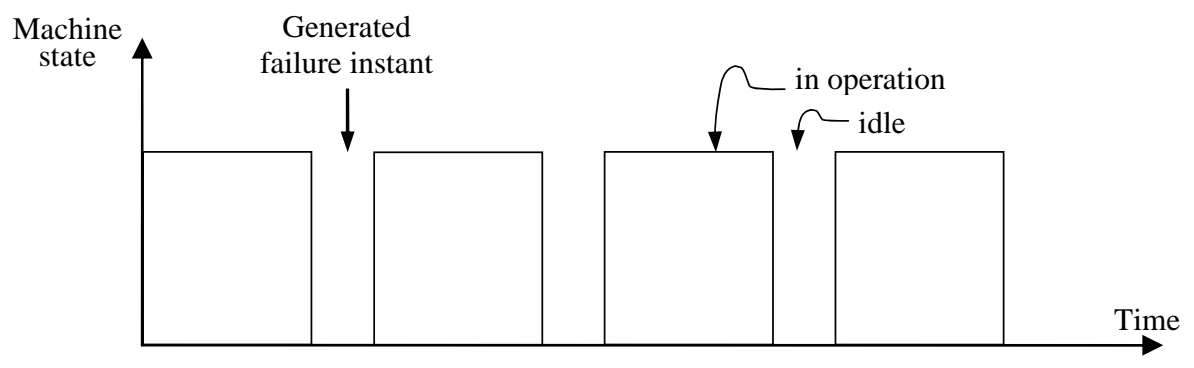

(a) Failure instant generated according to the machine life time distribution

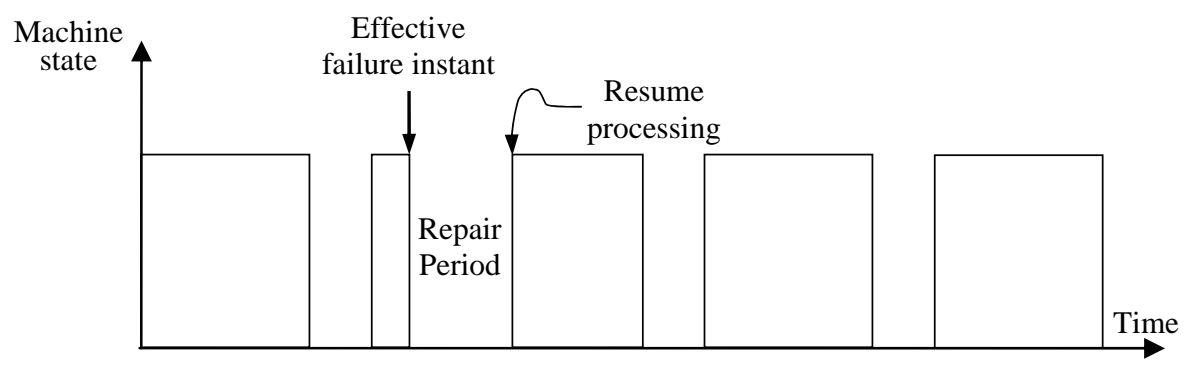

(b) Effective failure instant evaluated by the FAILURE INSTANT CORRECTION module

Figure 5: Failure instant correction according to the ODF mode.

\section{NUMERICAL RESULTS}

In this section, we compare the effective throughput of automatic transfer lines generated by simulation model to those generated by approximate models. Thousands of experiments on several transfer line configurations have been carried out in order to evaluate the performance of these models in assessing the throughput of unbuffered non-homogeneous transfer lines. In this study, we have considered lines with 2, 3, 4, 5, 6, 7, 8, 9, 10, 20, 30, 40, and 50 machines with different manufacturing times, failure and repair rates.

To analyse the effect of individual machine availability on the transfer line throughput, two ranges for machine availability are considered without loss of generality: low availability $\left(U T R_{i} \in[70,75 \%]\right)$, and high availability $\left(U T R_{i} \in[95,100 \%]\right)$.

In this research, and for each configuration, all transfer line parameters were generated randomly: bottleneck machine, machine processing times, machine mean times to failure and to repair. Table I gives ten randomly generated configurations for non-homogeneous production lines with four machines (with $U T R_{i} \in[70,75 \%]$ ).

Table I: Randomly generated configurations for a 4-machine line.

\begin{tabular}{|c|c|c|c|c|c|c|c|c|c|c|c|c|}
\hline \multirow{2}{*}{$\begin{array}{l}\text { Line } \\
\text { Config. }\end{array}$} & \multicolumn{3}{|c|}{ Machine 1} & \multicolumn{3}{|c|}{ Machine 2} & \multicolumn{3}{|c|}{ Machine 3} & \multicolumn{3}{|c|}{ Machine 4} \\
\hline & $T_{1}$ & MTTF $_{1}$ & MTTR $_{1}$ & $\mathbf{T}_{2}$ & $\mathrm{MTTF}_{2}$ & MTTR $_{2}$ & $T_{3}$ & $\mathrm{MTTF}_{3}$ & MTTR $_{3}$ & $T_{4}$ & $\mathrm{MTTF}_{4}$ & $\mathrm{MTTR}_{4}$ \\
\hline 1 & 19.35 & 673.27 & 261.83 & 18.8 & 258.44 & 95.59 & 14.49 & 738.62 & 301.69 & 17.88 & 143.02 & 55.62 \\
\hline 2 & 10.1 & 532.26 & 206.99 & 10.49 & 953.38 & 352.62 & 14.26 & 159.27 & 58.91 & 14.41 & 785.78 & 261.93 \\
\hline 3 & 12.62 & 751.1 & 263.9 & 9.85 & 215.55 & 92.38 & 12.24 & 343.2 & 147.09 & 11.97 & 650.7 & 265.78 \\
\hline 4 & 22.27 & 309.34 & 108.69 & 19.74 & 186.15 & 68.85 & 16.87 & 879.53 & 342.04 & 17.23 & 578.11 & 236.13 \\
\hline 5 & 10.48 & 808.31 & 346.42 & 11.4 & 487.95 & 189.76 & 10.39 & 496.68 & 174.51 & 8.79 & 408.37 & 166.8 \\
\hline 6 & 11.04 & 822.22 & 304.11 & 10.04 & 920.8 & 358.09 & 8.85 & 790.74 & 307.51 & 9.41 & 770.72 & 330.31 \\
\hline 7 & 15.59 & 852.41 & 348.17 & 17.91 & 278.73 & 113.85 & 18.66 & 229.91 & 80.78 & 19.22 & 879.73 & 325.38 \\
\hline 8 & 16.94 & 265.46 & 93.27 & 18.07 & 556.22 & 216.31 & 17.98 & 718.96 & 308.13 & 17.82 & 587.6 & 251.83 \\
\hline 9 & 15.76 & 844.76 & 328.52 & 12.08 & 713.24 & 250.6 & 13.35 & 774.87 & 332.09 & 11.09 & 177.71 & 65.73 \\
\hline 10 & 9.86 & 527.53 & 185.35 & 12.56 & 688.65 & 281.28 & 12.61 & 377.98 & 154.39 & 13.14 & 500.75 & 175.94 \\
\hline$\ldots$ & $\ldots$ & $\ldots$ & $\ldots$ & $\ldots$ & $\ldots$ & $\ldots$ & $\ldots$ & $\ldots$ & $\ldots$ & $\ldots$ & $\ldots$ & $\ldots$ \\
\hline
\end{tabular}


Subsequent to the experiments in Table I, Table II shows the throughput generated by the simulation model and by the techniques studied in this paper. It also gives the throughput relative error by comparing analytical results to the simulation ones according to Eq. (6).

$$
\text { Th_RE }(\%)=\frac{\overline{T h_{s_{-} c}}-T h_{T k_{-} c}}{\overline{T h_{s_{-} c}}} \cdot 100 \%
$$

where: $\quad c$ is the randomly generated $c^{\text {th }}$ configuration of an $n$-machine production line.

$\overline{T h_{s_{-} c}}$ - mean simulated throughput of the $c^{\text {th }}$ line configuration.

$T h_{T k_{-} c}$ - analytical throughput for the $c^{\text {th }}$ line configuration.

Table II: Comparison of simulation results with analytical results for production line configurations of Table I.

\begin{tabular}{|c|c|c|c||c|c|}
\hline \multirow{2}{*}{$\begin{array}{c}\text { Line } \\
\text { Config. }\end{array}$} & \multicolumn{2}{|c|}{ Throughput (parts/time unit) } & \multicolumn{2}{c|}{ Th_RE (\%) } \\
\cline { 2 - 6 } & Simul. & T1 & T2 & T1 & T2 \\
\hline $\mathbf{1}$ & 0.0214 & 0.0184 & 0.0202 & 14.28 & 5.72 \\
\hline $\mathbf{2}$ & 0.0310 & 0.0271 & 0.0282 & 12.42 & 9.04 \\
\hline $\mathbf{3}$ & 0.0318 & 0.0278 & 0.0303 & 12.33 & 4.65 \\
\hline $\mathbf{4}$ & 0.0196 & 0.0184 & 0.0178 & 5.95 & 9.08 \\
\hline $\mathbf{5}$ & 0.0363 & 0.0324 & 0.0340 & 10.67 & 6.19 \\
\hline $\mathbf{6}$ & 0.0379 & 0.0354 & 0.0352 & 6.53 & 7.15 \\
\hline $\mathbf{7}$ & 0.0214 & 0.0192 & 0.0205 & 10.08 & 4.23 \\
\hline $\mathbf{8}$ & 0.0216 & 0.0207 & 0.0213 & 4.14 & 1.33 \\
\hline $\mathbf{9}$ & 0.0278 & 0.0255 & 0.0250 & 8.24 & 10.09 \\
\hline $\mathbf{1 0}$ & 0.0317 & 0.0271 & 0.0302 & 14.75 & 4.85 \\
\hline $\mathbf{. .}$ & $\ldots$ & $\ldots$ & $\ldots$ & $\ldots$ & $\ldots$ \\
\hline
\end{tabular}

Fig. 6 shows the absolute value of the throughput mean relative errors (mean for 100 randomly generated configurations for each $n$-machine line) obtained by comparing results of each technique to simulation ones (Eq. 7).

$$
\text { Th_MRE (\%) }=\frac{\left|\sum_{c=1}^{100} \frac{\overline{T h_{s_{-}}}-T h_{T k_{-} c}}{\overline{T h_{s_{-} c}}}\right|}{100} \cdot 100 \%
$$

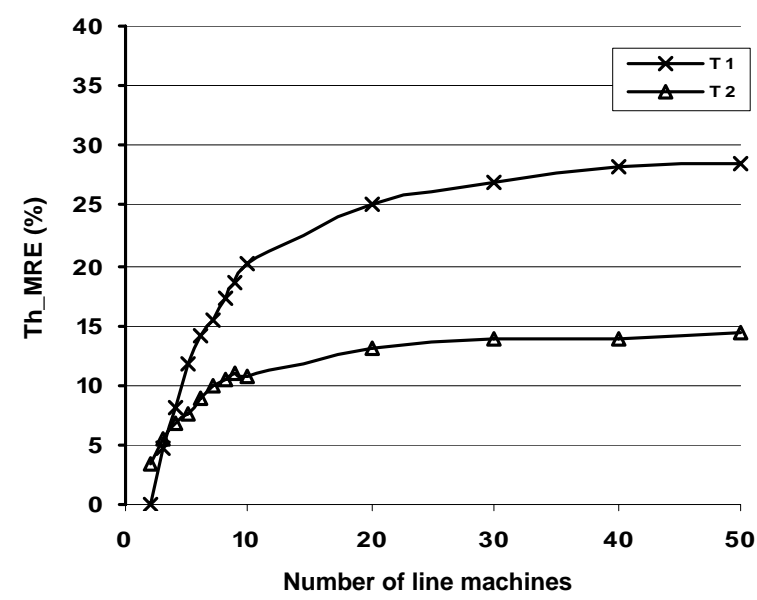

(a) Machines with $U T R_{i} \in[70,75 \%]$

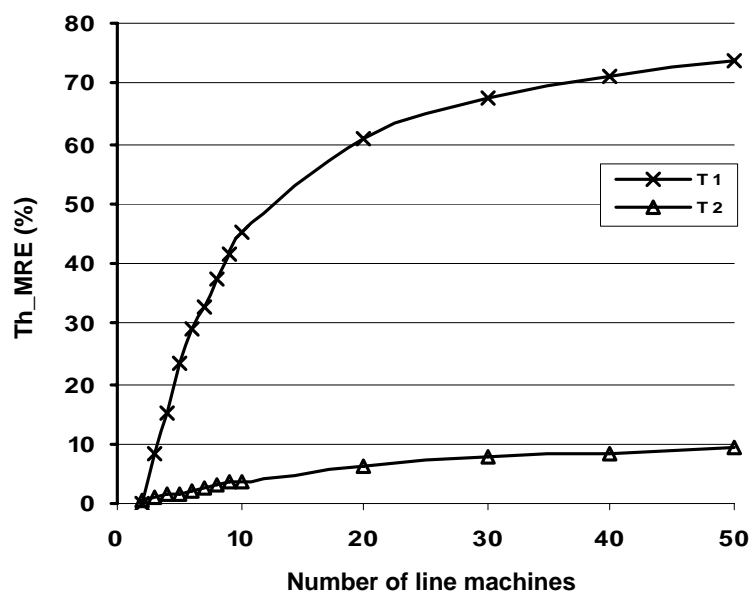

(b) Machines with $U T R_{i} \in[95,100 \%]$

Figure 6: Throughput mean relative error of studied techniques versus simulation. 
The analysis of Fig. 6 conducts to the following results:

1- When the transfer line has more than two machines, technique T2 outperforms generally technique $\mathrm{T} 1$.

2- Compared to simulation results, mean relative errors produced by techniques $\mathrm{T} 1$ and $\mathrm{T} 2$ grows with the number of line machines.

3- Despite behaviour of technique T2, the throughput relative error given by technique T1 increases according to individual machine availability.

4- Compared to simulation results, techniques T1 and T2 underestimate non-homogeneous transfer line throughput.

5- When the transfer line has two machines, technique T1 give an exact evaluation of the line throughput. Effectively, for the 2-machine transfer lines, the throughput relative error is negligible (Fig. 6) and all Student t-tests are accepted despite the individual machine availability range (Table III).

6- Table III shows that hypothesis $\mathrm{H}_{0}$ is rejected when the number of line machines increases. Theses results confirm that formulations ensuing from techniques $\mathrm{T} 1$ and $\mathrm{T} 2$ are not suitable to assess the throughput of unbuffered, non-homogeneous transfer lines.

Table III: Percentage of Successful Student Tests (for 100 randomly generated configurations for each $n$-machine line).

\begin{tabular}{|c|c|c|c|c|}
\hline \multirow{2}{*}{$\begin{array}{l}\text { Number of } \\
\text { Machines }\end{array}$} & \multicolumn{2}{|c|}{$U T R_{i} \in[70,75 \%]$} & \multicolumn{2}{|c|}{$U T R_{i} \in[95,100 \%]$} \\
\hline & T1 (\% succes) & T2 (\% succes) & T1 (\% succes) & T2 (\% succes) \\
\hline 2 & 100 & 10 & 100 & 31 \\
\hline 3 & 4 & 0 & 3 & 8 \\
\hline 4 & 0 & 0 & 0 & 4 \\
\hline 5 & 0 & 0 & 0 & 0 \\
\hline 6 & 0 & 0 & 0 & 0 \\
\hline 7 & 0 & 0 & 0 & 0 \\
\hline 8 & 0 & 0 & 0 & 0 \\
\hline 9 & 0 & 0 & 0 & 0 \\
\hline 10 & 0 & 0 & 0 & 0 \\
\hline 20 & 0 & 0 & 0 & 0 \\
\hline 30 & 0 & 0 & 0 & 0 \\
\hline 40 & 0 & 0 & 0 & 0 \\
\hline 50 & 0 & 0 & 0 & 0 \\
\hline
\end{tabular}

\section{CONCLUSIONS}

In this paper, we analyse the throughput of unreliable, non-homogeneous transfer lines with deterministic processing times and no storage buffers. A general discrete event simulation model was developed and it imitates the real dynamic and stochastic behaviour of such transfer lines.

The throughput of such transfer lines generated by the simulation model was compared to several approximation techniques dealing with throughput assessment of non-homogeneous transfer lines: techniques proposed by Gershwin [6], Dallery et al. [7], Liu and Buzacott [8], and Chen and Yuan [9].

Several transfer line configurations with different lengths and randomly generated machine processing times, failure and repair rates have been experimented (lines including from 2 to 50 machines). Simulation and analytical results show that the techniques proposed by Gershwin, Dallery et al., Liu and Buzacott, and Chen and Yuan conduct to significant 
errors. Statistical tests confirm that these techniques cannot give a good estimate of the throughput of unbuffered, non-homogeneous transfer lines, especially when the transfer line is composed of several machines. Even though, the trivial technique (proposed by Chen and Yuan) outperforms the other estimating techniques when the transfer line is composed of more than two machines.

This research showed the necessity to develop more accurate analytical models to assess the throughput of unbuffered, non-homogeneous transfer lines subject to operation dependant failures. Simulation should be used to validate such an analytical model. In the absence of such a model, simulation should be used to assess the throughput of unbuffered, nonhomogeneous transfer lines subject to operation dependant failures.

\section{REFERENCES}

[1] Papadopoulos, H. T.; Heavey, C. (1996). Queueing Theory in Manufacturing Systems Analysis and Design: A Classification of Models for Production and Transfer Lines, European Journal of Operational Research, Vol. 92, 1-27

[2] Buzacott, J. A.; Shanthikumar, J. G. (1993). Stochastic Models of Manufacturing Systems, Prentice-Hall, Inc., Englewood Cliffs, New Jersey, USA

[3] Gershwin, S. B. (1994). Manufacturing Systems Engineering, Prentice-Hall, New-York, USA

[4] Buzacott, J. A. (1968). Prediction of the Efficiency of Production Systems without Internal Storage, International Journal of Production Research, Vol. 6, No. 3, 173-188

[5] Dallery, Y.; Gershwin, S. B. (1992). Manufacturing Flow Line Systems: A Review of Models and Analytical Results, Queueing Systems Theory and Applications, Vol. 12, 3-94

[6] Gershwin, S. B. (1987). Representation and Analysis of Transfer Lines with Machines that Have Different Processing Rates, Annals of Operations Research, Vol. 9, 511-530

[7] Dallery, Y.; David, R.; Xie, X. L. (1989). Approximate Analysis of Transfer Lines with Unreliable Machines and Finite Buffers, IEEE Transactions on Automatic Control, Vol. 34, No. 9, 943-953

[8] Liu, X.-G.; Buzacott, J. A. (1990). Approximate Models of Assembly Systems with Finite Inventory Banks, European Journal of Operational Research, Vol. 45, 143-154

[9] Chen, C.-T.; Yuan, J. (2004). Transient Throughput Analysis for a Series Type System of Machines in Terms of Alternating Renewal Processes, European Journal of Operational Research, Vol. 155, 178-197

[10] Tan, B. (1997). Variance of the Throughput of an N-Station Production Line with No Intermediate Buffers and Time Dependent Failures, European Journal of Operational Research, Vol. 101, 560-576

[11] Barlow, R. E.; Proschan, F. (1975). Statistical Theory of Reliability and Life Testing, Holt Rinehart and Winston, New York, USA

[12] Gnedenko, B.; Ushakov, I. (1995). Probabilistic Reliability Engineering, John Wiley \& Sons, New York, USA

[13] Zimmern, B. (1956). Études de la Propagation des Arrêts Aléatoires dans les Chaînes de Production, Revue de Statistiques Appliquées, Vol. 4, 85-104

[14] Dhouib, K.; Gharbi, A.; Ayed, S. (2006). Disponibilité et Taux de Production des Lignes de Transfert Homogènes, Sans Stocks Intermédiaires, $4^{\text {th }}$ International JTEA4 Conference, Hammamet, Tunisia

[15] Pritsker, A. A.; O’Reilly, J. J. (1999). Simulation with Visual SLAM and AweSim, John Wiley \& Sons, New York, USA 